\title{
Differential Subordinations for Nonanalytic Functions
}

\author{
Georgia Irina Oros and Gheorghe Oros \\ Department of Mathematics, University of Oradea, Strada Universităţii, No. 1, 410087 Oradea, Romania \\ Correspondence should be addressed to Georgia Irina Oros; georgia_oros_ro@yahoo.co.uk
}

Received 1 April 2014; Accepted 25 May 2014; Published 18 June 2014

Academic Editor: V. Ravichandran

Copyright (c) 2014 G. I. Oros and G. Oros. This is an open access article distributed under the Creative Commons Attribution License, which permits unrestricted use, distribution, and reproduction in any medium, provided the original work is properly cited.

In the paper by Mocanu (1980), Mocanu has obtained sufficient conditions for a function in the classes $C^{1}(U)$, respectively, and $C^{2}(U)$ to be univalent and to map $U$ onto a domain which is starlike (with respect to origin), respectively, and convex. Those conditions are similar to those in the analytic case. In the paper by Mocanu (1981), Mocanu has obtained sufficient conditions of univalency for complex functions in the class $C^{1}$ which are also similar to those in the analytic case. Having those papers as inspiration, we try to introduce the notion of subordination for nonanalytic functions of classes $C^{1}$ and $C^{2}$ following the classical theory of differential subordination for analytic functions introduced by Miller and Mocanu in their papers (1978 and 1981) and developed in their book (2000). Let $\Omega$ be any set in the complex plane $\mathbb{C}$, let $p$ be a nonanalytic function in the unit disc $U, p \in C^{2}(U)$, and let $\psi(r, s, t ; z): \mathbb{C}^{3} \times U \rightarrow \mathbb{C}$. In this paper, we consider the problem of determining properties of the function $p$, nonanalytic in the unit disc $U$, such that $p$ satisfies the differential subordination $\psi\left(p(z), D p(z), D^{2} p(z)-D p(z) ; z\right) \subset \Omega \Rightarrow p(U) \subset \Delta$.

\section{Introduction and Preliminaries}

Let $U=\{z \in \mathbb{C}:|z|<1\}$ be the unit disc of the complex plane with $\bar{U}=\{z \in \mathbb{C}:|z| \leq 1\}$ and $\partial U=\{z \in \mathbb{C}:|z|=1\}$, and let $H(U)$ be the class of analytic functions on $U$. We also consider the class of nonanalytic functions of classes $C^{1}$ and $C^{2}$, respectively.

Definition 1 (see $[1,2])$. Let $f$ be a complex function defined in the unit $\operatorname{disc} U$.

For $z=x+i y \in U$, we put

$$
f(z)=u(x, y)+i v(x, y) .
$$

We say that the function $f$ belongs to the class $C^{1}(U)$ or $C^{2}(U)$, if the functions $u=\operatorname{Re} f$ and $v=\operatorname{Im} f$ of the real variables $x$ and $y$ have continuous first-order or second-order partial derivatives in $U$, respectively.

For $f \in C^{1}(U)$, we denote

$$
\begin{aligned}
& D f=z \cdot \frac{\partial f}{\partial z}-\bar{z} \cdot \frac{\partial f}{\partial \bar{z}}, \\
& D f=z \cdot \frac{\partial f}{\partial z}+\bar{z} \cdot \frac{\partial f}{\partial \bar{z}},
\end{aligned}
$$

where

$$
\begin{aligned}
& \frac{\partial f}{\partial z}=\frac{1}{2}\left(\frac{\partial f}{\partial x}-i \frac{\partial f}{\partial y}\right), \\
& \frac{\partial f}{\partial \bar{z}}=\frac{1}{2}\left(\frac{\partial f}{\partial x}+i \frac{\partial f}{\partial y}\right) .
\end{aligned}
$$

Remark 2. (1) It is obvious that the linear differential operators $D$ and $\mathscr{D}$ verify the usual rules of differential calculus. For instance,

$$
\begin{gathered}
D(f g)=f D g+g D f, \\
\mathscr{D}(f g)=f \mathscr{D} g+g \mathscr{D} f, \\
D\left(\frac{f}{g}\right)=\frac{g D f-f D g}{g^{2}}, \\
\mathscr{D}\left(\frac{f}{g}\right)=\frac{g \mathscr{D} f-f \mathscr{D} g}{g^{2}}, \\
D(f \circ g)=\frac{\partial f}{\partial g} D g+\frac{\partial f}{\partial \bar{g}} D \bar{g},
\end{gathered}
$$




$$
\begin{gathered}
\mathscr{D}(f \circ g)=\frac{\partial f}{\partial g} \mathscr{D} g+\frac{\partial f}{\partial \bar{g}} \mathscr{D} \bar{g} \\
D(D f)=D^{2} f, \quad \mathscr{D}(\mathscr{D} f)=\mathscr{D}^{2} f .
\end{gathered}
$$

(2) We also have the following useful formulas:

$$
\begin{gathered}
D \bar{f}=-\overline{D f}, \quad \mathscr{D} \bar{f}=\overline{D f}, \\
D \operatorname{Re} f=i \operatorname{Im} D f, \quad \mathscr{D} \operatorname{Re} f=\operatorname{Re} D f, \\
D \operatorname{Im} f=-i \operatorname{Re} D f, \quad \mathscr{D} \operatorname{Im} f=\operatorname{Im} \mathscr{D} f, \\
D|f|=i|f| \operatorname{Im} \frac{D f}{f}, \quad \mathscr{D}|f|=|f| \operatorname{Re} \frac{\mathscr{D} f}{f}, \\
D \arg f=-\operatorname{Re} \frac{D f}{f}, \quad f(z) \neq 0, \\
\mathscr{D} \arg f=\operatorname{Im} \frac{\mathscr{D} f}{f}, \quad f(z) \neq 0 .
\end{gathered}
$$

(3) If $h \in C^{1}(\mathbb{R})$, then $D h(z \bar{z})=0$ and $\mathscr{D h}(\arg z)=0$.

Hence the constant functions for the operators $D$ and $\mathscr{D}$ are the functions of the forms $h(z \bar{z})$ and $h(\arg z)$, respectively.

(4) If $z=r e^{i \theta}$, we have

$$
\text { (a) } \frac{\partial f}{\partial \theta}=i D f, \quad \frac{\partial f}{\partial r}=\frac{1}{r} \mathscr{D} f \text {. }
$$

Therefore,

$$
\begin{aligned}
& \text { (b) } \frac{\partial|f|}{\partial \theta}=-|f| \operatorname{Im} \frac{D f}{f}, \quad \frac{\partial|f|}{\partial r}=\frac{|f|}{r} \operatorname{Re} \frac{\mathscr{D} f}{f}, \\
& \text { (c) } \frac{\partial}{\partial \theta} \arg f=\operatorname{Re} \frac{D f}{f}, \quad \frac{\partial}{\partial \theta} \arg f=\frac{1}{r} \operatorname{Im} \frac{\mathscr{D} f}{f} .
\end{aligned}
$$

(5) The Jacobian of the function $f \in C^{1}(U)$ is given by

$$
J f=\left|\frac{\partial f}{\partial z}\right|^{2}-\left|\frac{\partial f}{\partial \bar{z}}\right|^{2} .
$$

If $J f(z)>0$, for $z \in U$, then it is well-known that $f$ is a locally homeomorphic function preserving the orientation.

(6) Consider

$$
\operatorname{Re}[D f \overline{\mathscr{D f}}]=|z|^{2} J f
$$

Let

$M_{1}(U)=\left\{f: U \longrightarrow \mathbb{C}\right.$, nonanalytic in $\left.U, f \in C^{1}(U)\right\}$,

$M_{2}(U)=\left\{f: U \longrightarrow \mathbb{C}\right.$, nonanalytic in $\left.U, f \in C^{2}(U)\right\}$,

and let $\mathscr{H}(U)$ denote the class of analytic functions in $U$.

\section{Main Results}

We will give the definition of subordination for nonanalytic functions of classes $C^{1}$ and $C^{2}$ following the classical theory of differential subordination introduced by S. S. Miller and P. T. Mocanu in papers $[3,4]$ and developed in their book [5].

Definition 3. Let $f$ and $g$ be two nonanalytic functions with $f \in C^{1}(U)$ and $g \in C^{1}(U)$. One says that the function $f$ is subordinated to the function $g$, denoted by $f \prec g$ or $f(z) \prec g(z)$, if there exists a function $w$ nonanalytic in $U$, $w \in C^{1}(U)$ with $w(0)=0$ and $|w(z)|<1, z \in U$, such that $f(z)=g(w(z)), z \in U$.

Property 1. If $f \prec g$, then $f(0)=g(0)$ and $f(U) \subseteq g(U)$.

Proof. From Definition 3 we have $w(0)=0$ and $|w(z)|<1$ and using Lemma C (Section 4) we have $|w(z)| \leq|z|, \quad z \in U$.

Then

$$
\begin{aligned}
f(U) & =\{g(w(z)): z \in U\} \\
& =\{g(\zeta):|\zeta| \leq|z|, z \in U\} \subseteq g(U) .
\end{aligned}
$$

For the case when the function $g$ is nonanalytic and injective with $g \in C^{1}(U)$ the following theorem holds.

Theorem 4. Let $f$ and $g$ be two nonanalytic functions in $U$ with $f \in C^{1}(U)$ and $g \in C^{1}(U)$ and $g$ injective in $U$.

Then $f \prec g$ if and only if $f(0)=g(0)$ and $f(U) \subset g(U)$.

Proof. The first part of Theorem 4 is given by Property 1 .

Let us assume $f(0)=g(0)$ and $f(U) \subseteq g(U)$.

We let $g(U)=\Delta$ and since $g$ is injective, there exists the inverse function $g^{-1}: \Delta \rightarrow U$. Then function $w(z)=$ $g^{-1}[f(z)]$ is a nonanalytic function in $U$ with $w \in C^{1}(U)$ and verifies evidently the conditions $w(0)=0,|w(z)|<1, z \in U$. From $w(z)=g^{-1}[f(z)]$, we obtain

$$
g(w(z))=g\left[g^{-1}(f(z))\right]=f(z)
$$

which gives that $f \prec g$.

We next present the general form of the method of differential subordinations (or the method of admissible functions) for nonanalytic functions.

Let $\Omega$ and $\Delta$ be any sets in $\mathbb{C}$, let $p$ be nonanalytic in the unit disc $U, p \in C^{2}(U), p(0)=a, a \in \mathbb{C}$, and let $\psi(r, s, t ; z)$ : $\mathbb{C}^{3} \times U \rightarrow \mathbb{C}, \psi \in C^{1}(U)$.

This theory deals with generalizations of the following implication:

$(\alpha) \quad\left\{\psi\left(p(z), D p(z), D^{2} p(z)-D p(z)\right): z \in U\right\} \subset \Omega$

$$
\Longrightarrow p(U) \subset \Delta \text {. }
$$

Remark 5. If $p \in \mathscr{H}(U)$, then relation $(\alpha)$ is equivalent to

$$
\left\{\psi\left(p(z), z p^{\prime}(z), z^{2} p^{\prime \prime}(z)\right): z \in U\right\} \subset \Omega \Longrightarrow p(U) \subset \Delta
$$


which is the general form of the implications from the classical theory of subordinations.

Related to condition $(\alpha)$, we state three problems that characterize the theory of differential subordinations for nonanalytic functions in the unit disc.

Problem 1. Given $\Omega$ and $\Delta$, find conditions on $\psi$ such that $(\alpha)$ holds. We call such a $\psi$ an admissible function.

Problem 2. Given $\psi$ and $\Omega$, find $\Delta$ such that $(\alpha)$ holds. Furthermore, find the "smallest" such $\Delta$.

Problem 3. Given $\psi$ and $\Delta$, find $\Omega$ such that $(\alpha)$ holds. Furthermore, find the "largest" such $\Omega$.

If either $\Omega$ or $\Delta$ in $(\alpha)$ is a simply connected domain, then $(\alpha)$ can be rewritten in terms of subordination.

Let $\Delta$ be a simply connected domain containing the point $a$ and $\Delta \neq \mathbb{C}$. Let $q, q \in C^{2}(U)$, be a nonanalytic function which is a conformal mapping of $U$ onto $\Delta$ such that $q(0)=a$.

In this case, $(\alpha)$ can be rewritten as

$$
\begin{aligned}
\left(\alpha^{\prime}\right) \quad & \left\{\psi\left(p(z), D p(z), D^{2} p(z)-D p(z)\right): z \in U\right\} \subset \Omega \\
& \Longrightarrow p(z) \prec q(z) .
\end{aligned}
$$

If $\Omega$ is also a simply connected domain and $\Omega \neq \mathbb{C}$, then there is a function $h, h \in C^{2}(U)$, nonanalytic, which is a conformal mapping of $U$ onto $\Omega$, such that $h(0)=\psi(a, 0,0 ; 0)$. If, in addition, the function $\psi\left(p(z), D p(z), D^{2} p(z)-D p(z)\right)$ is a nonanalytic function in $U, \psi \in C^{1}(U)$, then $(\alpha)$ can be rewritten as

$$
\begin{aligned}
\left(\alpha^{\prime \prime}\right) \quad \psi( & \left(z(z), D p(z), D^{2} p(z)-D p(z) ; z\right) \prec h(z) \\
& \Longrightarrow p(z) \prec q(z)
\end{aligned}
$$

This last result leads us to some important definitions.

Definition 6. Let $\psi: \mathbb{C}^{3} \times U \rightarrow \mathbb{C}$ be a nonanalytic function in $U, \psi \in C^{1}(U)$, and let $h$ be a nonanalytic function in $U$, $h \in C^{1}(U)$.

If $p$ is a nonanalytic function in $U, p \in C^{2}(U)$, and satisfies the (second-order) differential subordination

$$
(\beta) \quad \psi\left(p(z), D p(z), D^{2} p(z)-D p(z) ; z\right) \prec h(z),
$$

then $p$ is called a solution of the differential subordination.

The nonanalytic function $g \in C^{2}(U)$ is called a dominant of the solutions of the differential subordination or more simply a dominant, if $p \prec q$ for all $p$ satisfying $(\beta)$. A dominant $\tilde{q}$ that satisfies $\tilde{q} \prec q$ for all dominants $q$ of $(\beta)$ is said to be the best dominant of $(\beta)$. Note that the best dominant is unique up to a rotation of $U$.

Let $\Omega$ be a set in $\mathbb{C}$ and suppose that $(\beta)$ is replaced by

$$
\left(\beta^{\prime}\right) \quad \psi\left(p(z), D p(z), D^{2} p(z)-D p(z) ; z\right) \in \Omega
$$

Although this is a differential inclusion, we will also refer to $\left(\beta^{\prime}\right)$ as a (second-order) differential subordination and use the same definitions of solution, dominant, and best dominant as given above.

In the case when $\Omega$ and $\Delta$ in $(\alpha)$ are simply connected domains, we have seen that $(\alpha)$ can be rewritten in terms of subordinations, such as given in $\left(\alpha^{\prime \prime}\right)$. Using this and Definition 6, we can restate Problems 1-3 as follows.

Problem $1^{\prime}$. Given $h$ and $q$ two nonanalytic and injective functions in $U, h, q \in C^{1}(U)$, find a class of admissible functions $\psi[h, q]$ such that $\left(\alpha^{\prime \prime}\right)$ holds.

Problem $2^{\prime}$. Given the differential subordination $\left(\alpha^{\prime \prime}\right)$, find a dominant $q$. Moreover, find the best dominant.

Problem $3^{\prime}$. Given $\psi, \psi \in C^{1}(U)$, nonanalytic function in $U$, and dominant $q, q \in C^{1}(U)$, nonanalytic function in $U$, find the largest class of nonanalytic and injective functions $h$ in $U$, $h \in C^{1}(U)$, such that $\left(\alpha^{\prime \prime}\right)$ holds.

Definition 7. One lets $Q$ denote the set of functions

$$
q(z)=u(x, y)+i v(x, y)
$$

nonanalytic and injective on $\bar{U} \backslash E(q)$ with $q \in C^{1}(U)$, where

$$
E(q)=\left\{\zeta \in \partial U: \lim _{z \rightarrow \zeta} q(z)=\infty\right\} .
$$

Moreover, we assume that $D q(\zeta) \neq 0$, for $\zeta \in \partial U \backslash E(q)$. The set $E(q)$ is called exception set.

The functions $q_{1}(z)=\bar{z}, q_{2}(z)=1+\bar{z}$, and $q_{3}(z)=$ $(1+\bar{z}) /(1-\bar{z})$ are in $Q$; hence, $Q$ is a nonempty set.

\section{The Class of Admissible Functions for Nonanalytic Functions}

Definition 8. Let $\Omega \subset \mathbb{C}$, let $q$ be a nonanalytic function, $q \in$ $Q, q \in C^{2}(U)$, and let $n$ be a natural number, $n \geq 1$. One lets $\Psi_{n}[\Omega, q]$ denote the class of functions $\psi: \mathbb{C}^{3} \times U \rightarrow \mathbb{C}$ which satisfy the condition

(A) $\quad \psi(r, s, t ; z) \notin \Omega$, when $r=q(\zeta), s=m D(\zeta)$,

$$
\operatorname{Re}\left(\frac{t}{s}+1\right) \geq m \operatorname{Re} \frac{D^{2} q(\zeta)}{D q(\zeta)},
$$

where $z \in U, \zeta \in \partial U \backslash E(q)$, and $m \geq n \geq 1$.

The set $\Psi_{n}[\Omega, q]$ is called the class of admissible functions and condition $(A)$ is called admissibility condition.

Remark 9. (1) If $\psi: \mathbb{C}^{2} \times U \rightarrow U$, the admissibility condition becomes

$$
\left(A^{\prime}\right) \quad \psi(r, s ; z) \notin \Omega, \quad \text { when } r=q(\zeta), s=m D(\zeta),
$$

for $z \in U$. where $z \in U, \zeta \in \partial U \backslash E(q)$, and $m \geq n \geq 1$. 
(2) If $\psi: \mathbb{C} \times U \rightarrow \mathbb{C}$, the admissibility condition becomes

$$
\left(A^{\prime \prime}\right) \quad \psi(r ; z) \notin \Omega, \quad \text { when } r=q(\zeta),
$$

where $z \in U, \zeta \in \partial U \backslash E(q)$, and $m \geq n \geq 1$.

(3) We let $\Psi_{1}[\Omega, q]=\Psi[\Omega, q]$.

Remark 10. If $g \in \mathscr{H}(U)$, then the admissibility condition $(A)$ becomes the well-known admissibility condition for analytic functions $\psi(r, s, t ; z) \notin \Omega$, when $r=q(\zeta), s=m D q(\zeta)=$ $m \zeta q^{\prime}(\zeta)$, and

$$
\begin{aligned}
\operatorname{Re}\left(\frac{t}{s}+1\right) & \geq m \operatorname{Re} \frac{D^{2} q(\zeta)}{D q(\zeta)} \\
& =m \operatorname{Re} \frac{\zeta^{2} q^{\prime \prime}(\zeta)+\zeta q^{\prime}(\zeta)}{\zeta q^{\prime}(\zeta)} \\
& =m \operatorname{Re}\left[\frac{\zeta q^{\prime \prime}(\zeta)}{q^{\prime}(\zeta)}+1\right] .
\end{aligned}
$$

\section{Fundamental Lemmas}

In order to prove the fundamental theorems, we must first prove some auxiliary theorems.

Lemma A. Let $f$ be a nonconstant continuous injective function inside a circle of center $z_{0}$ and arbitrary radius, with $f\left(z_{0}\right) \neq 0$. Then, inside this circle, there exist points $z^{\prime}$ and $z^{\prime \prime}$ such that

$$
\left|f\left(z^{\prime}\right)\right|<\left|f\left(z_{0}\right)\right|<\left|f\left(z^{\prime \prime}\right)\right| \text {. }
$$

Proof. If function $f$ is continuous inside the circle $C\left(z_{0}, r\right)$, then function $|f|$ is also continuous inside this circle. Then there exists a neighbourhood $C\left(z_{0}, \varepsilon\right)$ such that $|f|$ is a bounded function. Then there exist two points $z^{\prime}$ and $z^{\prime \prime}$ inside $C\left(z_{0}, \varepsilon\right)$ where function $|f|$ has a smallest value and a highest value, $m$ and $M$, respectively, $m=f\left(z^{\prime}\right)$ and $M=$ $f\left(z^{\prime \prime}\right)$. Then, for any $z \in C\left(z_{0}, \varepsilon\right)$, we have

$$
\left|f\left(z^{\prime}\right)\right|<|f(z)|<\left|f\left(z^{\prime \prime}\right)\right| \text {. }
$$

Lemma B (maximum of modulus). Let $f$ be a nonconstant, continuous, injective function inside a circle $\bar{C}\left(z_{0}, R\right)=\{z \in$ $\left.\mathbb{C}:\left|z-z_{0}\right| \leq R\right\}$. Then $|f|$ cannot attain its maximum value inside the circle, meaning

$$
\max _{z \in \bar{C}\left(z_{0}, R\right)}|f(z)|=\max _{z \in \partial C\left(z_{0}, R\right)}|f(z)| .
$$

Proof. Assume that there exists a point $z_{1}$ inside $C\left(z_{0}, R\right)$ such that $\max _{z \in C\left(z_{0}, R\right)}|f(z)|=\left|f\left(z_{1}\right)\right|$. We have $f\left(z_{1}\right) \neq 0$ since if $|f(z)|=0$ the function would be constant $f(z) \equiv 0$. If function $|f|$ is continuous inside $\bar{C}\left(z_{0}, R\right)$, then from Lemma A we have that there exists a point $z^{\prime \prime}$ inside $C\left(z_{0}, R\right)$ such that $\left|f\left(z^{\prime \prime}\right)\right|>\left|f\left(z^{\prime}\right)\right|$. This is impossible since we have assumed that $\left|f\left(z_{1}\right)\right|$ is the maximum value. Hence, $z^{\prime \prime}$ belongs to the border of the circle.
Lemma C (the first part of Schwarz's lemma). Let $f$ be a continuous function in $U=\{z \in \mathbb{C}:|z|<1\}$ and let the following conditions hold: $f(0)=0$ and $|f(z)|<1$; then $|f(z)| \leq|z|$, for all $z \in U$.

Proof. Let the function

$$
g(z)= \begin{cases}\frac{f(z)}{z} & \text { if } z \in \bar{U}-\{0\} \\ \lim _{z \rightarrow 0} \frac{f(z)}{z} & \text { if } z=0\end{cases}
$$

Since functions $f(z)$ and $h(z)=z$ are continuous in $\bar{U}$, we have that $f(z) / z$ is continuous in $\bar{U}-\{0\}$.

From $g(0)=\lim _{z \rightarrow 0} f(z) / z$, we obtain that $g(z)$ is continuous in $\bar{U}$.

Let $z \in U$ with $|z|=r<1$. Then function $g(z)$ is continuous in $\bar{C}(0, r)$. Using Lemma $B$, we have

$$
\begin{aligned}
\max _{z \in \bar{C}(0, r)}|g(z)| & =\max _{z \in \partial C(0, r)}|g(z)|=\max _{z \in \partial C(0, r)}\left|\frac{f(z)}{z}\right| \\
& =\max _{z \in \partial C(0, r)} \frac{|f(z)|}{|z|} \leq \frac{1}{r} \quad \forall z \in \bar{C}(0, r) .
\end{aligned}
$$

If we let $r \rightarrow 1$, we obtain $|q(z)| \leq 1$ which implies $|f(z)| \leq|z|$.

Remark 11. Lemmas A, B, and C hold also for some continuous, nonanalytic functions.

We assume that Lemma A and Lemma B also hold without the condition that function $f$ is injective, but this condition helped in giving the strict inequalities.

Lemma 12 (I. S. Jack, S. S. Miller, and P. T. Mocanu). Let $z_{0}=$ $r_{0} e^{i \theta_{0}}$ with $0<r_{0}<1$ and let $f$ be a nonanalytic function in $U$,

$$
f(z)=u(x, y)+i v(x, y),
$$

continuous on $\bar{U}\left(0, r_{0}\right)$ with $f(z) \not \equiv 0$ and $f \in C^{2}(U)$ (or $\left.f \in C^{1}(U)\right)$.

If

$$
\left|f\left(z_{0}\right)\right|=\max \left\{|f(z)|: z \in \bar{U}\left(0, r_{0}\right)\right\}
$$

then there exists a number $m \in \mathbb{R}, m \geq n \geq 1$, such that

(i) $\operatorname{Re} D f\left(z_{0}\right) / f\left(z_{0}\right)=m$,

(ii) $\operatorname{Re} D^{2} f\left(z_{0}\right) / D f\left(z_{0}\right) \geq m$.

Proof. Let $z=r e^{i \theta}, 0<r<1$ and $\theta \in[0,2 \pi)$. Then

$$
f(z)=R(\theta) e^{i \phi(\theta)} .
$$

By differentiating (32) with respect to $\theta$, we obtain

$$
\frac{\partial f(z) / \partial \theta}{f(z)}=\frac{\partial R(\theta)}{\partial \theta}+i \frac{\partial \phi(\theta)}{\partial \theta} .
$$


In [1], the author proved that if $z=r e^{i \phi}$, then

$$
\frac{\partial f(z)}{\partial \theta}=i D f(z)
$$

Using (34) in (33), we obtain

$$
\frac{i D f(z)}{f(z)}=\frac{R^{\prime}(\theta)}{R(\theta)}+i \phi^{\prime}(\theta) .
$$

For $z=z_{0}$, (35) becomes

$$
\frac{i D f\left(z_{0}\right)}{f\left(z_{0}\right)}=\frac{R^{\prime}\left(\theta_{0}\right)}{R\left(\theta_{0}\right)}+i \phi^{\prime}\left(\theta_{0}\right) .
$$

Since $f(z) \not \equiv 0$ and $\theta_{0}$ is a point of maximum for $R(\theta)$, we have $R^{\prime}\left(\theta_{0}\right)=0$ and $R^{\prime \prime}\left(\theta_{0}\right) \leq 0$.

From (36), we have

$$
i \frac{D f\left(z_{0}\right)}{f\left(z_{0}\right)}=i \phi^{\prime}\left(\theta_{0}\right)
$$

and we deduce

$$
\frac{D f\left(z_{0}\right)}{f\left(z_{0}\right)}=\phi^{\prime}\left(\theta_{0}\right)=m, \quad m \in \mathbb{R} .
$$

We show that $m>0$. In order to prove this, we define the function

$$
g(z)= \begin{cases}\lim _{z \rightarrow z_{0}} \frac{f\left(z_{0} z\right)}{f\left(z_{0}\right)} \cdot \frac{1}{z^{n-1}} & \text { if } z=0 \\ \frac{f\left(z_{0} z\right)}{f\left(z_{0}\right)} \cdot \frac{1}{z^{n-1}} & \text { if } z \in \bar{U}(0,1) \backslash\{0\}, n \geq 1 .\end{cases}
$$

Since functions $f(z)$ and $h(z)=z$ are continuous in $\bar{U}$, function $f(z) / z$ is continuous in $\bar{U}(0,1)-\{0\}$. From the definition of function $g$,

$$
g(0)=\lim _{z \rightarrow z_{0}} \frac{f\left(z_{0} z\right)}{f\left(z_{0}\right)} \cdot \frac{1}{z^{n-1}},
$$

and hence function $g$ is continuous in $\bar{U}$.

Using Lemma $B$, we deduce

$$
|g(z)| \leq \max \left\{\left|\frac{f\left(z_{0} z\right)}{f\left(z_{0}\right) z^{n-1}}\right|:|z|=1\right\}=1, \quad \forall z \in \bar{U} .
$$

Using Lemma C, we have $|g(z)| \leq z, z \in \bar{U}$, since

$$
\left|\frac{f\left(z_{0} z\right)}{f\left(z_{0}\right)}\right| \leq|z|^{n} \text {. }
$$

If we take $z=r, 0 \leq r<1$, in (42), we obtain

$$
\left|\frac{f\left(z_{0} z\right)}{f\left(z_{0}\right)}\right| \leq r^{n}
$$

and, from this,

$$
\operatorname{Re} \frac{f\left(z_{0} r\right)}{f\left(z_{0}\right)} \leq r^{n}
$$

We calculate

$$
\begin{aligned}
\frac{d}{d r}\left(\frac{f\left(z_{0} r\right)}{f\left(z_{0}\right)}\right) & =\lim _{\substack{r \rightarrow 1 \\
r<1}} \frac{f\left(z_{0} r\right)-f\left(z_{0}\right)}{(r-1) f\left(z_{0}\right)} \\
& =\lim _{\substack{r \rightarrow 1 \\
r<1}}\left[\left(1-\frac{f\left(z_{0} r\right)}{f\left(z_{0}\right)}\right) \frac{1}{1-r}\right] .
\end{aligned}
$$

Since $m \in \mathbb{R}$, we deduce that

$$
\begin{aligned}
m & =\lim _{\substack{r \rightarrow 1 \\
r<1}}\left[\left(1-\operatorname{Re} \frac{f\left(z_{0} r\right)}{f\left(z_{0}\right)}\right) \frac{1}{1-r}\right] \\
& \geq \lim _{\substack{r \rightarrow 1 \\
r<1}}\left(1-r^{n}\right) \frac{1}{1-r} \\
& =\lim _{\substack{r \rightarrow 1 \\
r<1}} \frac{(1-r)\left(1+r+\cdots+r^{n-1}\right)}{1-r}=n,
\end{aligned}
$$

and hence $m \geq n \geq 1$. In order to prove inequality (ii), we differentiate relation (35) with respect to $\theta$ and we obtain

$$
\begin{aligned}
& \frac{f(\partial / \partial \theta)(D f)-D f(\partial / \partial \theta)(f)}{f^{2}} \\
& \quad=-i \frac{R^{\prime \prime}(\theta) R(\theta)-R^{\prime}(\theta) R^{\prime}(\theta)}{R^{2}(\theta)}+\phi^{\prime \prime}(\theta) .
\end{aligned}
$$

Using $\partial f / \partial \theta=i D f$ and $D(D f)=D^{2} f$, (47) becomes

$$
\begin{aligned}
& \frac{i f \cdot D^{2} f-i(D f)^{2}}{f^{2}} \\
& \quad=-i \frac{R^{\prime \prime}(\theta) R(\theta)-\left[R^{\prime}(\theta)\right]^{2}}{R^{2}(\theta)}+\phi^{\prime \prime}(\theta) .
\end{aligned}
$$

For $z=z_{0}$, (48) becomes

$$
\begin{aligned}
& \frac{\text { if }\left(z_{0}\right) D^{2} f\left(z_{0}\right)-i\left[D f\left(z_{0}\right)\right]^{2}}{f^{2}\left(z_{0}\right)} \\
& =-i \frac{R^{\prime \prime}\left(\theta_{0}\right) R(\theta)-\left[R^{\prime}\left(\theta_{0}\right)\right]^{2}}{R^{2}\left(\theta_{0}\right)}+\phi^{\prime \prime}(\theta) .
\end{aligned}
$$

Since $f(z) \not \equiv 0$ and $\theta_{0}$ is a point of maximum for $R(\theta)$, we have $R^{\prime}\left(\theta_{0}\right)=0$ and $R^{\prime \prime}\left(\theta_{0}\right)<0$, and from (49) we have

$$
i \frac{f\left(z_{0}\right) D^{2} f\left(z_{0}\right)-\left[D f\left(z_{0}\right)\right]^{2}}{f^{2}\left(z_{0}\right)}=-i \frac{R^{\prime \prime}\left(\theta_{0}\right)}{R\left(\theta_{0}\right)}+\phi^{\prime \prime}\left(\theta_{0}\right)
$$

and we get

$$
\begin{gathered}
i\left\{\frac{D^{2} f\left(z_{0}\right)}{D f\left(z_{0}\right)} \cdot \frac{D f\left(z_{0}\right)}{f\left(z_{0}\right)}-\left[\frac{D f\left(z_{0}\right)}{f\left(z_{0}\right)}\right]\right\}^{2} \\
\quad=-i \frac{R^{\prime \prime}\left(\theta_{0}\right)}{R\left(\theta_{0}\right)}+\phi^{\prime \prime}\left(\theta_{0}\right) .
\end{gathered}
$$


Since $D f\left(z_{0}\right) / f\left(z_{0}\right)=m$, (51) becomes

$$
i\left[\frac{D^{2} f\left(z_{0}\right)}{D f\left(z_{0}\right)} m-m^{2}\right]=-i \frac{R^{\prime \prime}\left(\theta_{0}\right)}{R\left(\theta_{0}\right)}+\phi^{\prime \prime}\left(\theta_{0}\right) .
$$

Relation (52) can be written in the form

$$
\begin{aligned}
i m & {\left[\operatorname{Re} \frac{D^{2} f\left(z_{0}\right)}{D f\left(z_{0}\right)}-m+i \operatorname{Im} \frac{D^{2} f\left(z_{0}\right)}{D f\left(z_{0}\right)}\right] } \\
& =-i \frac{R^{\prime \prime}\left(\theta_{0}\right)}{R\left(\theta_{0}\right)}+\phi^{\prime \prime}\left(\theta_{0}\right)
\end{aligned}
$$

and we deduce

$$
m\left[\operatorname{Re} \frac{D^{2} f\left(z_{0}\right)}{D f\left(z_{0}\right)}-m\right]=-\frac{R^{\prime \prime}\left(\theta_{0}\right)}{R\left(\theta_{0}\right)} \geq 0,
$$

and hence

$$
\operatorname{Re} \frac{D^{2} f\left(z_{0}\right)}{D f\left(z_{0}\right)}-m \geq 0
$$

Remark 13. If $f \in \mathscr{H}(U)$ then we have

(i) $z_{0} f^{\prime}\left(z_{0}\right) / f\left(z_{0}\right)=m$;

(ii) $\operatorname{Re}\left[\left(z_{0} f^{\prime \prime}\left(z_{0}\right) / f\left(z_{0}\right)\right)+1\right] \geq m$.

Lemma 14 (S. S. Miller and P. T. Mocanu). Let $q \in Q$ with $q(0)=a$, and let $p$ be a nonanalytic function $p \in C(U)$ or $p \in C^{2}(U)$ with $p(z) \not \equiv a, n \geq 1$.

If there exist points $z_{0} \in U$ and $\zeta_{0} \in \partial U \backslash E(q)$ such that $p\left(z_{0}\right)=q\left(\zeta_{0}\right)$ and $p\left(\bar{U}\left(0, r_{0}\right)\right) \subset q(\bar{U}) \backslash E(q)$, where $r_{0}=\left|z_{0}\right|$, then there exists a real number $m, m \geq n$, such that

$$
\operatorname{Re} \frac{D^{2} p\left(z_{0}\right)}{D p\left(z_{0}\right)} \geq m \operatorname{Re} \frac{D^{2} q\left(\zeta_{0}\right)}{D q\left(\zeta_{0}\right)} .
$$

Proof. Let the function

$$
f(z)=q^{-1}(p(z)), \quad z \in \bar{U}\left(0, r_{0}\right) .
$$

For $z=z_{0}$, we have

$$
f\left(z_{0}\right)=q^{-1}\left(p\left(z_{0}\right)\right) .
$$

From the hypothesis, we have that $p\left(z_{0}\right)=q\left(\zeta_{0}\right)$ and, using this in (58), we obtain

$$
f\left(z_{0}\right)=q^{-1}\left(p\left(z_{0}\right)\right)=q^{-1}\left(q\left(\zeta_{0}\right)\right)=\zeta_{0} .
$$

Using relation (59), we get

$$
\left|f\left(z_{0}\right)\right|=\left|\zeta_{0}\right|=1 .
$$

From the maximum of modulus theorem, we have that

$$
|f(z)| \leq 1, \quad z \in \bar{U}\left(0, r_{0}\right) .
$$

On the other hand, $f(0)=q^{-1}(p(0))=q^{-1}(a)=q^{-1}(q(0))=$ $a$, since $q(0)=a$. Using Lemma 12 , we have

$$
\frac{D f\left(z_{0}\right)}{f\left(z_{0}\right)}=m
$$

$$
\operatorname{Re} \frac{D^{2} f\left(z_{0}\right)}{D f\left(z_{0}\right)} \geq m
$$

Using (57), we obtain

$$
q(f(z))=q\left(q^{-1}(p(z))\right)=p(z), \quad z \in \bar{U}\left(0, r_{0}\right)
$$

and we have

$$
p(z)=q(f(z)), \quad z \in \bar{U}\left(0, r_{0}\right) .
$$

If $z=r e^{i \theta}, 0 \leq r \leq 1$ and $\theta \in[0,2 \pi]$, then (65) becomes

$$
p\left(r e^{i \theta}\right)=q\left(f\left(r e^{i \theta}\right)\right), \quad \theta \in[0,2 \pi] .
$$

By differentiating relation (66) with respect to $\theta$, we obtain

$$
\frac{\partial}{\partial \theta} p\left(r e^{i \theta}\right)=\frac{\partial}{\partial \theta} q\left(f\left(r e^{i \theta}\right)\right) \cdot \frac{\partial}{\partial \theta} f\left(r e^{i \theta}\right) .
$$

Using (a), we have

$$
\begin{aligned}
& \frac{\partial}{\partial \theta} p(z)=i D p(z), \\
& \frac{\partial}{\partial \theta} q(f(z))=i D q(f(z)), \\
& \frac{\partial}{\partial \theta} f(z)=i D f(z) .
\end{aligned}
$$

Using (68) in (67), we have

$$
D p(z)=i D q(f(z)) D f(z) .
$$

Let

$$
f(z)=\zeta
$$

Using (70) in (69), we obtain

$$
D p(z)=i D q(\zeta) \cdot \zeta \cdot \frac{D f(z)}{f(z)} .
$$

For $z=z_{0}$, (71) becomes

$$
D p\left(z_{0}\right)=i \zeta_{0} \cdot D q\left(\zeta_{0}\right) \cdot \frac{D f\left(z_{0}\right)}{f\left(z_{0}\right)} .
$$

Using (62) in (72), we obtain

$$
D p\left(z_{0}\right)=i m \zeta_{0} D q\left(\zeta_{0}\right) .
$$

By differentiating relation (69), with respect to $\theta$, we obtain

$$
\begin{aligned}
& \frac{\partial}{\partial \theta} D p(z) \\
& \quad=i\left[\frac{\partial}{\partial \theta} D q(f(z)) D f(z)+D q(f(z)) \frac{\partial}{\partial \theta} D f(z)\right] .
\end{aligned}
$$


Using $(\alpha)$, we have

$$
D^{2} p(z)=i\left[D^{2} q(f(z)) D f(z)+D q(f(z)) D^{2} f(z)\right]
$$

which is equivalent to

$$
\frac{D^{2} p(z)}{D p(z)}=-i \cdot \frac{D^{2} q(f(z)) D f(z)+D q(f(z)) D^{2} f(z)}{D p(z)} \text {. }
$$

From (72), for $m=1$ and $D p\left(z_{0}\right)=i \zeta_{0} D q\left(\zeta_{0}\right)$, we have

$$
\frac{D^{2} p\left(z_{0}\right)}{D p\left(z_{0}\right)}=\frac{D^{2} q\left(f\left(z_{0}\right)\right) D f\left(z_{0}\right)+D q\left(f\left(z_{0}\right)\right) D^{2} f\left(z_{0}\right)}{\zeta_{0} D q\left(\zeta_{0}\right)} \text {. }
$$

From (59), we have $\zeta_{0}=f\left(z_{0}\right)$ and we obtain

$$
\begin{aligned}
\frac{D^{2} p\left(z_{0}\right)}{D p\left(z_{0}\right)}= & \frac{D^{2} q\left(f\left(z_{0}\right)\right) D f\left(z_{0}\right)}{D q\left(f\left(z_{0}\right)\right) f\left(z_{0}\right)} \\
& +\frac{D q\left(f\left(z_{0}\right)\right) D^{2} f\left(z_{0}\right)}{D q\left(f\left(z_{0}\right)\right) f\left(z_{0}\right)}
\end{aligned}
$$

which is equivalent to

$$
\begin{aligned}
\frac{D^{2} p\left(z_{0}\right)}{D p\left(z_{0}\right)}= & \frac{D^{2} q\left(f\left(z_{0}\right)\right)}{D q\left(f\left(z_{0}\right)\right)} \cdot \frac{D f\left(z_{0}\right)}{f\left(z_{0}\right)} \\
& +\frac{D^{2} f\left(z_{0}\right)}{D f\left(z_{0}\right)} \cdot \frac{D f\left(z_{0}\right)}{f\left(z_{0}\right)} .
\end{aligned}
$$

Using (62), we have

$$
\begin{aligned}
\frac{D^{2} p\left(z_{0}\right)}{D p\left(z_{0}\right)}= & m \cdot \frac{D^{2} q f\left(z_{0}\right)}{D q\left(f\left(z_{0}\right)\right)} \\
& +m \cdot \frac{D^{2} f\left(z_{0}\right)}{D f\left(z_{0}\right)} .
\end{aligned}
$$

Using (63), we obtain

$$
\begin{aligned}
\operatorname{Re} & \frac{D^{2} p\left(z_{0}\right)}{D p\left(z_{0}\right)} \\
& =m\left[\operatorname{Re} \frac{D^{2} q\left(f\left(z_{0}\right)\right)}{D q\left(f\left(z_{0}\right)\right)}+\operatorname{Re} \frac{D^{2} f\left(z_{0}\right)}{D f\left(z_{0}\right)}\right] \\
& \geq m\left[\operatorname{Re} \frac{D^{2} q\left(f^{2}\left(z_{0}\right)\right)}{D q\left(f\left(z_{0}\right)\right)}+m\right] \geq m \operatorname{Re} \frac{D^{2} q\left(f\left(z_{0}\right)\right)}{D q\left(f\left(z_{0}\right)\right)} .
\end{aligned}
$$

Since $f\left(z_{0}\right)=\zeta_{0}$, from (71), we obtain

$$
\operatorname{Re} \frac{D^{2} p\left(z_{0}\right)}{D p\left(z_{0}\right)} \geq m \operatorname{Re} \frac{D^{2} q\left(\zeta_{0}\right)}{D q\left(\zeta_{0}\right)} .
$$

Remark 15. If $f \in \mathscr{H}(U)$, then $\operatorname{Re}\left(\left(z_{0} p^{\prime \prime}\left(z_{0}\right) / p^{\prime}\left(z_{0}\right)\right)+1\right) \geq$ $m \operatorname{Re}\left(\left(\zeta_{0} q^{\prime \prime}\left(\zeta_{0}\right) / q^{\prime}\left(\zeta_{0}\right)\right)+1\right)$

Lemma 16 (S. S. Miller and P. T. Mocanu). Let $q$ be $a$ nonanalytic function, $q \in Q$, with $q(0)=a$, let $p$ be a nonanalytic function with $p(z) \not \equiv a$ and $p \in C^{1}(U)$, and let $n$ be a natural number with $n \geq 1$. If $p(z)$ is not subordinate to $q(z)$, written as $p(z) \nless q(z)$, then there exist points $z_{0}=r_{0} e^{i \theta_{0}}$ and $\zeta_{0} \in \partial U \backslash E(q)$ and a number $m \geq n \geq 1$ such that $p\left(U\left(0, r_{0}\right)\right) \subset q(U)$ and

(i) $p\left(z_{0}\right)=q\left(\zeta_{0}\right)$;

(ii) $\operatorname{Re} D^{2} p\left(z_{0}\right) / D p\left(z_{0}\right) \geq m \operatorname{Re}\left(D^{2} q\left(\zeta_{0}\right) / D q\left(\zeta_{0}\right)\right)$.

Proof. From the hypothesis, we have that $p(0)=q(0)=a$ and since $p(z) \nprec q(z)$, we have $p(U) \not \subset q(U)$.

Let $r_{0}=\sup \{r: p(U(0, r)) \subset q(U)\}$.

Since $p(U) \not \subset q(U)$, for $0<r_{0}<1$, we have $p\left(U\left(0, r_{0}\right)\right) \subset$ $q(U)$ and $p\left(\bar{U}\left(0, r_{0}\right)\right) \subset q(U)$. We get that there exists a point $z_{0} \in \partial U\left(0, r_{0}\right)$ such that $p\left(z_{0}\right) \in \partial q(U)$. From this, we obtain that there exists $\zeta_{0} \in \partial U \backslash E(q)$ such that $p\left(z_{0}\right)=q\left(\zeta_{0}\right)$.

We conclude that there exist $z_{0} \in U$ and $\zeta_{0} \in \partial U \backslash E(q)$ such that $p\left(z_{0}\right)=q\left(\zeta_{0}\right)$ and $p\left(U\left(0, r_{0}\right)\right) \subset q(U)$. From Lemma 14, we have

$$
\operatorname{Re} \frac{D^{2} p\left(z_{0}\right)}{D p\left(z_{0}\right)} \geq m \operatorname{Re} \frac{D^{2} q\left(\zeta_{0}\right)}{D q\left(\zeta_{0}\right)}
$$

which concludes the proof.

\section{Fundamental Theorems}

Theorem 17. Let $\psi \in \Psi_{n}[\Omega, q], q$ a nonanalytic function, $q \in C^{2}(U)$ with $q(0)=a$ and $q(U)=\Delta$. If function $p$ is $a$ nonanalytic function, $p \in C^{2}(U)$ with $p(0)=a$, and verifies the condition

$$
\psi\left(p(z), D p(z), D^{2} p(z)-D p(z) ; z\right) \in \Omega, \quad z \in U,
$$

then $p(z) \prec q(z)$.

Proof. We assume that $p(z) \nprec q(z)$. From Lemma 16, we have that there exist points $z_{0} \in U$ and $\zeta_{0} \in \partial U \backslash E(q)$ and number $m \geq n$ such that the following conditions are satisfied:

(i) $p\left(z_{0}\right)=q\left(\zeta_{0}\right)$;

(ii) $\operatorname{Re}\left(D^{2} p\left(z_{0}\right) / D p\left(z_{0}\right)\right) \geq m \operatorname{Re}\left(D^{2} q\left(\zeta_{0}\right) / D q\left(\zeta_{0}\right)\right)$.

Using these conditions with $r=p\left(z_{0}\right), s=D p\left(z_{0}\right), t=$ $D^{2} p\left(z_{0}\right)-D p\left(z_{0}\right)$, and $z=z_{0}$ in Definition 8 , we obtain

$$
\psi\left(p\left(z_{0}\right), D p\left(z_{0}\right), D^{2} p\left(z_{0}\right)-D\left(p\left(z_{0}\right)\right) ; z_{0}\right) \notin \Omega \text {. }
$$

Since (85) contradicts (84), we have that the assumption made is false; hence $p(z) \prec q(z), z \in U$.

Remark 18. Theorem 17 also holds if (84) is replaced by

$$
\begin{gathered}
\psi\left(p(w(z)), D(w(z)), D^{2}(w(z))-D(w(z)) ;\right. \\
w(z)) \subset \Omega, \quad z \in U,
\end{gathered}
$$

for any nonanalytic function $w$ which maps $U$ onto $U$. 
Remark 19. In the hypothesis of Theorem 17, we have assumed that the behaviour of $q$ is known on the border of $\Delta$. If we do not know the behaviour of $q$ on the border of $\Delta$, then we may also prove that $p(z) \prec q(z)$ using the following limit procedure.

Theorem 20. Let $\Omega \subset \mathbb{C}$, let $q$ be a nonanalytic function, $q \in$ $C^{2}(U)$, injective in $\bar{U}$, with $q(0)=a$, and let $\psi \in \Psi_{n}\left[\Omega, q_{\rho}\right]$ for some $\rho \in(0,1)$, where $q_{\rho}(z)=q(\rho z)$. If $p$ is a nonanalytic function, $p \in C^{2}(U)$ with $p(0)=a$, then

$$
\left\{\psi\left(p(z), D p(z), D^{2} p(z)-D p(z)\right): z \in U\right\} \subset \Omega
$$

implies that $p(z) \prec q(z)$.

Proof. Since $q_{\rho}(z)=q(\rho z)$, we have that the function $q_{\rho}$ is injective on $\bar{U}$; hence $E\left(q_{\rho}\right)=\phi$ and $q_{\rho} \in Q$. The function $\psi \in \Psi_{n}\left[\Omega, q_{\rho}\right]$ is an admissible function and

$$
\left\{\psi\left(p(z), D p(z), D^{2} p(z)-D p(z)\right): z \in U\right\} \subset \Omega,
$$

so, from Theorem 17, we have that

$$
p(z) \prec q(z) .
$$

On the other hand, $q_{\rho}(z)=q(\rho z)$ implies that

$$
q_{\rho}(z) \prec q(z)
$$

From (89) and (90), we obtain $p(z) \prec q_{\rho}(z) \prec q(z)$ which gives $p(z) \prec q(z)$.

Remark 21. In the case when $\Omega \subset \mathbb{C}, \Omega \neq \mathbb{C}$ is a simply connected domain and $h$ is a nonanalytic function, $h \in$ $C^{l}(U)$, and is injective in $U$; if we assume that $h(U)=\Omega$, then, by letting $\Psi_{n}[h, q] \equiv \Psi_{n}[h(U), q]$, from Theorem 17, we obtain the following result.

Theorem 22. Let $h$ be a nonanalytic function, injective in $U$, $h \in C^{1}(U)$ and $h(0)=a$ with $h(U)=\Omega$; let $q$ be a nonanalytic function, injective in $U, q \in C^{2}(U), q(0)=a$, and $q(U)=\Delta$. If $p$ is a nonanalytic function, injective in $U, p \in C^{2}(U)$ with $p(0)=a$, and function $\psi\left(p(z), D p(z), D^{2} p(z)-D p(z)\right)$ is a nonanalytic function, $\psi \in C^{1}(U)$ and $\psi(a, 0,0 ; 0)=h(0)=a$, then

$$
\psi\left(p(z), D p(z), D^{2} p(z)-D p(z)\right) \prec h(z)
$$

implies that

$$
p(z) \prec q(z), \quad z \in U
$$

This result can be extended for the case when the behaviour of $q$ on $\partial U$ is not known.

Theorem 23. Let $f$ and $q$ be nonanalytic functions, injective in $U, h \in C^{2}(U)$ with $h(U)=\Omega$ and $q \in C^{2}(U)$ with $q(0)=a$, $q(U)=\Delta$.
We let $h_{\rho}(z)=h(\rho z)$ and $q_{\rho}(z)=q(\rho z)$. Let function $\psi: \mathbb{C}^{3} \times U \rightarrow U$ be nonanalytic in $U, \psi \in C^{1}(U)$, with $\psi(a, 0,0 ; 0)=a$ and satisfying one of the following conditions:

(i) $\psi \in \Psi_{n}\left[h, q_{\rho}\right]$ for some $\rho \in(0,1)$ or

(ii) there exists a certain $\rho_{0} \in(0,1)$ such that $\psi \in$ $\Psi_{n}\left[h_{\rho}, q_{\rho}\right]$ for all $\rho \in\left(\rho_{0}, 1\right)$.

If function $p$ is nonanalytic in $U, p \in C^{2}(U)$, and function $\psi\left(p(z), D p(z), D^{2} p(z)-D p(z) ; z\right)$ is nonanalytic and injective in $U$, then

$$
\psi\left(p(z), D p(z), D^{2} p(z)-D p(z) ; z\right) \prec h(z)
$$

implies $p(z) \prec q(z)$.

Proof. (i) From Theorem 17, we have $p(z) \prec q_{\rho}(z)$. On the other hand, $q_{\rho}(z) \prec q(z)$ for $\rho \in(0,1)$. From $p(z) \prec q_{\rho}(z) \prec$ $q(z)$, we have that $p(z) \prec g(z), z \in U$.

(ii) If we let $p_{\rho}(z)=p(\rho z)$, then

$$
\begin{gathered}
\psi\left(p_{\rho}(z), D p_{\rho}(z), D^{2} p_{\rho}(z)-D p_{\rho}(z) ; \rho z\right) \\
=\psi\left(p(\rho z), D p(\rho z), D^{2} p(\rho z)-D p(\rho z) ;\right. \\
\rho(z)) \in h_{\rho}(U) .
\end{gathered}
$$

By applying Theorem 17 and Remark 18 with $w(z)=\rho z$, we obtain $p_{\rho}(z) \prec q_{\rho}(z)$ for all $\rho \in\left(\rho_{0}, 1\right)$.

By letting $\rho \rightarrow 1$, we obtain $p(z) \prec q(z)$.

Example 24. Let the function $p$ be nonanalytic in $U$; then

$$
p(z)+D^{2} p(z) \prec 5 M \frac{z}{\bar{z}}, \quad M>0 \Longrightarrow p(z) \prec M \frac{z}{\bar{z}} .
$$

Proof. Let the function $\psi: \mathbb{C}^{3} \times U \rightarrow \mathbb{C}$ be a nonanalytic function in $U, \psi \in C^{2}(U)$. In order to prove implication, it is enough to prove that $\psi \in \Psi_{n}[h, q]$. For that, it suffices to show that $\psi$ satisfies the admissibility condition.

Let $h(z)=5 M(z / \bar{z}), h(U)=U(0,5 M), q(z)=M(z / \bar{z})$, and $\psi(r, s, t)=r+s+t$.

In order to check the admissibility condition, we calculate

$$
\begin{aligned}
D q(z) & =z \frac{\partial}{\partial z}\left(M \frac{z}{\bar{z}}\right)-\bar{z} \cdot \frac{\partial}{\partial \bar{z}}\left(m \frac{z}{\bar{z}}\right) \\
& =M \frac{z}{\bar{z}}+M \overline{z z}=2 M \frac{z}{\bar{z}}, \\
D^{2} q(z) & =D(D q(z)) \\
& =z \cdot \frac{\partial}{\partial z}\left(2 M \frac{z}{\bar{z}}\right)-\bar{z} \cdot \frac{\partial}{\partial z}\left(2 M \frac{z}{\bar{z}}\right) \\
& =2 M \frac{z}{\bar{z}}+2 M \frac{z}{\bar{z}}=4 M \frac{z}{\bar{z}} .
\end{aligned}
$$


For $r=q(\zeta)=M \cdot(\zeta / \bar{\zeta})=M \cdot\left(\zeta^{2} / \zeta \cdot \bar{\zeta}\right)=M \zeta^{2}$, since $\zeta \cdot \bar{\zeta}=|\zeta|=1$,

$$
\begin{gathered}
s=m D q(\zeta)=m \cdot 2 M \cdot \frac{\zeta}{\bar{\zeta}}=2 m M \cdot \frac{\zeta \cdot \zeta}{\bar{\zeta} \cdot \zeta}=2 m M \zeta^{2}, \\
\operatorname{Re} \frac{D^{2} q(\zeta)}{D q(\zeta)}=2 .
\end{gathered}
$$

We check the admissibility condition:

$$
\begin{aligned}
|\psi(r, s, t)| & =|r+s+t|=\left|M \zeta^{2}+2 m M \zeta^{2}+t\right| \\
& =M\left|1+2 m+\frac{t}{M \zeta^{2}}\right|=M\left|1+2 m+\frac{2 m t}{2 m \zeta^{2} M}\right| \\
& =M\left|1+2 m\left(1+\frac{t}{s}\right)\right| \geq M \operatorname{Re}\left[1+2 m\left(1+\frac{t}{s}\right)\right] \\
& \geq M\left[1+2 m \operatorname{Re}\left(1+\frac{t}{s}\right)\right] \geq M\left[1+4 m^{2}\right] \geq 5 M,
\end{aligned}
$$

since $m \geq 1$. Hence, $\psi(r, s, t) \notin h(U) \Rightarrow \psi \in \Psi_{n}[h, q]$.

From Theorem 17, we have $p(z) \prec M \cdot(z / \bar{z})$.

\section{Conflict of Interests}

The authors declare that there is no conflict of interests regarding the publication of this paper.

\section{References}

[1] P. T. Mocanu, "Starlikeness and convexity for non-analytic functions in the unit disc," Mathematica, vol. 22, no. 1, pp. 77-83, 1980.

[2] P. T. Mocanu, "Sufficient conditions of univalency for complex functions in the class C1," Revue d'Analyse Numerique et de Theorie de l'Approximation, vol. 10, no. 1, pp. 75-79, 1981.

[3] S. S. Miller and P. T. Mocanu, "Second order differential inequalities in the complex plane," Journal of Mathematical Analysis and Applications, vol. 65, pp. 298-305, 1978.

[4] S. S. Miller and P. T. Mocanu, "Differential subordinations and univalent functions," Michigan Mathematical Journal, vol. 28, pp. 157-171, 1981.

[5] S. S. Miller and P. T. Mocanu, Differential Subordination. Theory and Applications, Marcel Dekker, New York, NY, USA, 2000. 


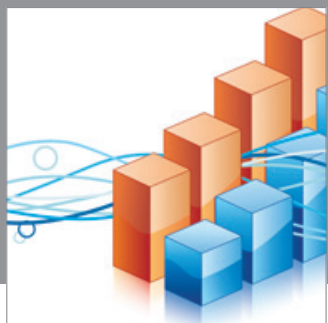

Advances in

Operations Research

mansans

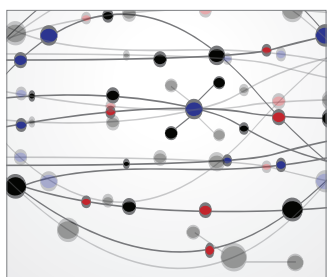

The Scientific World Journal
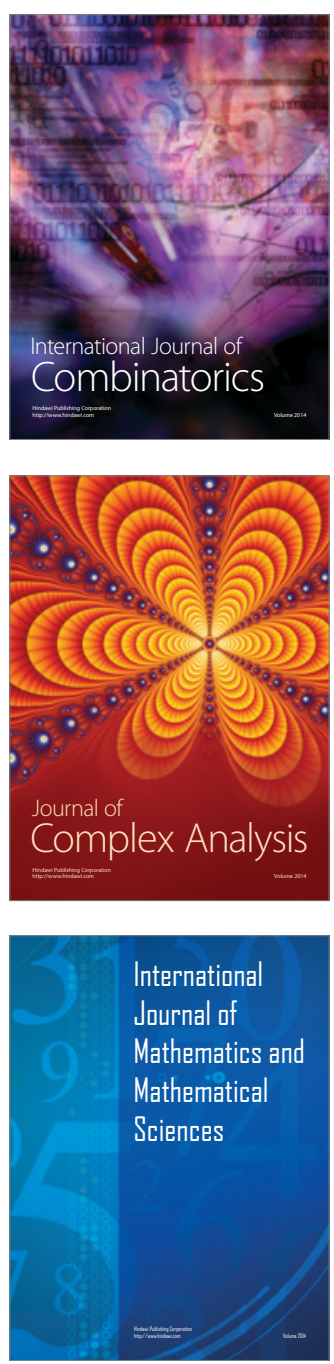
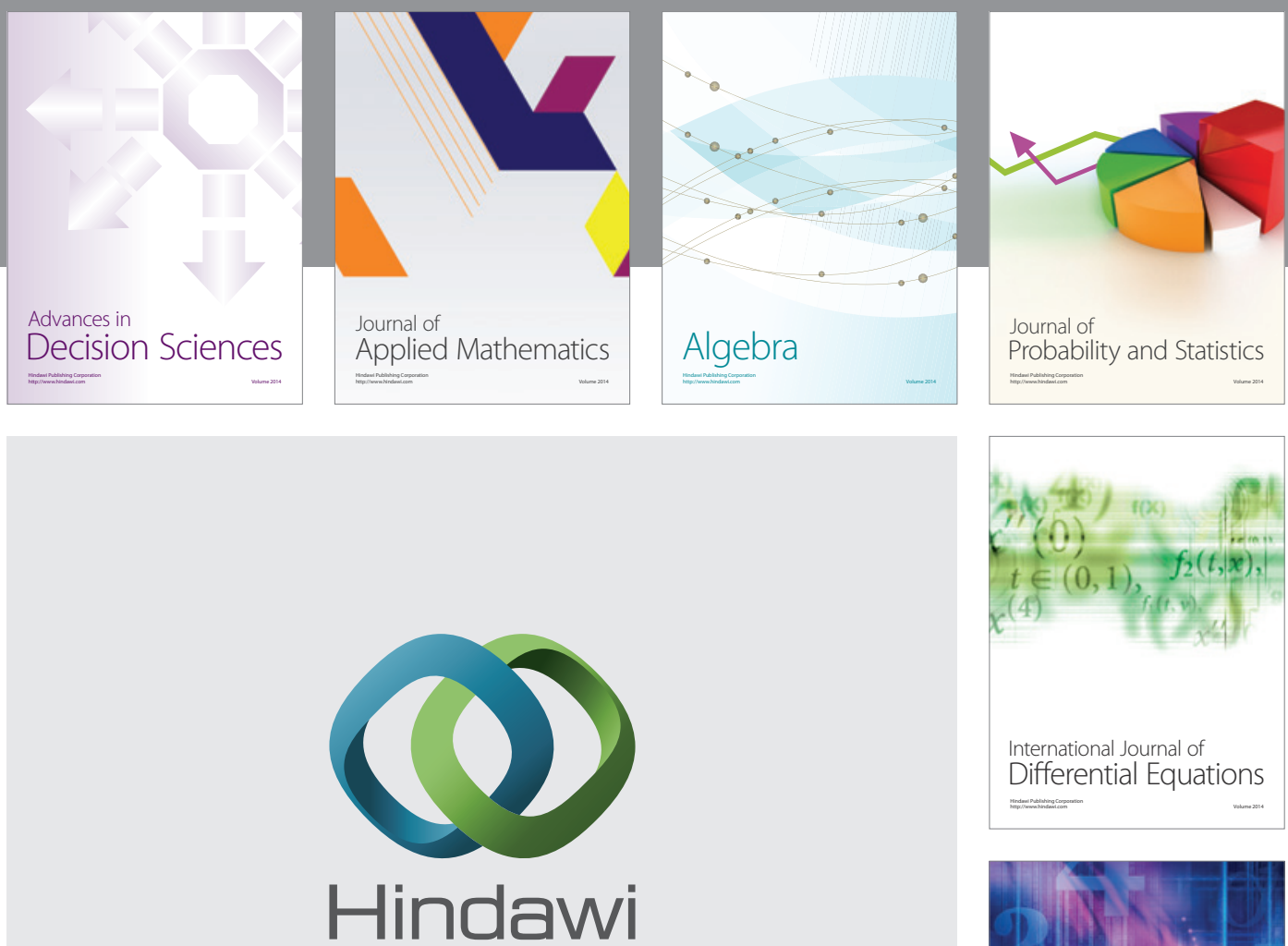

Submit your manuscripts at http://www.hindawi.com
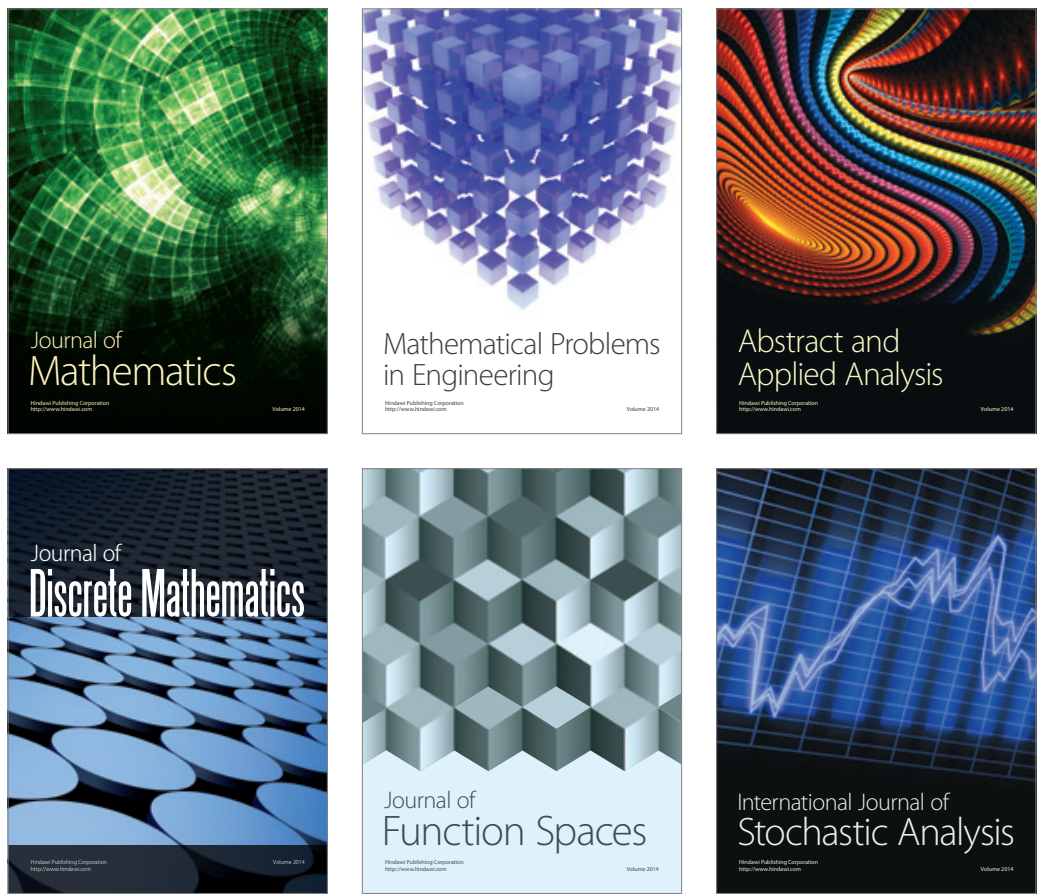

Journal of

Function Spaces

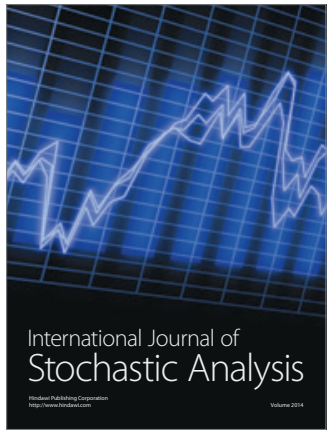

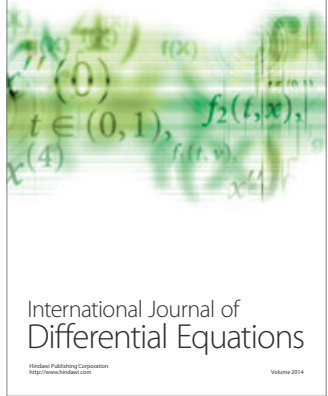
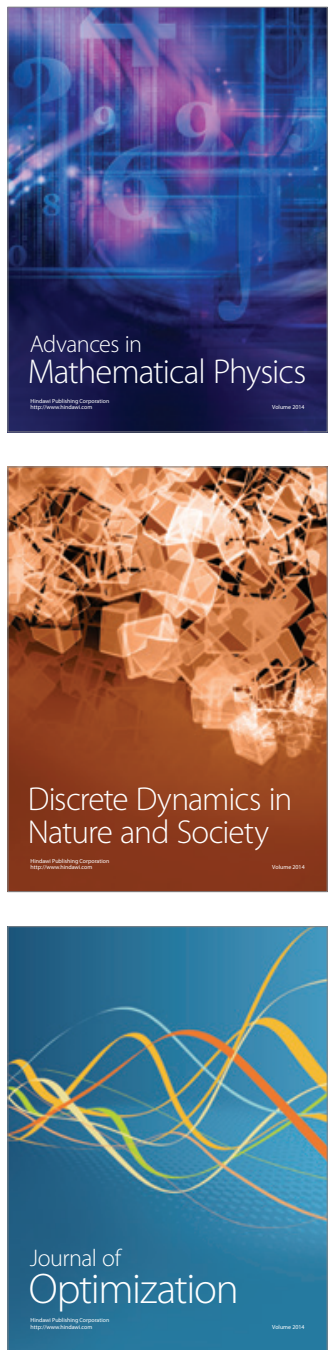